\title{
Beam Energy Dependent Calibration of STEM and BSE Detectors for Thin Film Thickness Estimation
}

\section{Radim Skoupy and Vladislav Krzyzanek}

The Czech Academy of Sciences, Institute of Scientific Instruments, Brno, Jihomoravsky kraj, Czech Republic

The well-established technique of quantitative scanning transmission electron microscopy (STEM) measurements uses a comparison of captured and normalized images with computer simulation of a sample. For each imaging session, it is necessary to calibrate the STEM detector to the used primary beam energy, probe current and contrast/brightness settings of the SEM that is simple due to the STEM detector position inside the SEM chamber (Figure 1 left). Local features like mass, thickness or composition can be estimated by this technique for thin - electron transparent - samples [1]. The principle of this technique is in general usable also for back-scatter electrons (BSE) with the possibilities for estimation of layer thickness on a substrate [2,3]. There are several methods for measuring thin film thickness on substrates by using different interacting probes like X-rays (X-ray reflectivity), light (modulation-based structuredillumination microscopy, ellipsometry, interferometry), ions (Rutherford back-scattering spectrometry, particle-induced X-ray emission, heavy ion elastic recoil detection) or mechanical contact (profilometry, atomic force microscopy) [4]. The focused electrons are used in the case of calibrated SEM-EDX [5], FIB-SEM cross-sections or TEM lamella analysis. Unfortunately, most of them damage the measured sample or give an average value from a large area of the sample.

The technique of quantitative imaging as described for STEM [1] is usable for BSE detectors too, but there occurs a problem with precise calibration and response measurement of the BSE detector due to its position in a SEM chamber, because primary electrons cannot hit the BSE detector directly as in the case of STEM. Most of the studies use a comparison of captured image with image of known standard sample for its normalization. We present a simple method for standard-less BSE detector calibration which together with Monte Carlo simulation of BSE signal emitted from a sample with given geometry brings a straight forward methodology of measuring the thickness of cover thin layer on substrates [6]. The inability to irradiate the BSE detector by primary electron beam directly is overcome by the application of an electron mirror for reversion of electron trajectories as shown in Figure 1 right. Resulting selfie image of the BSE detector gives detector response to the full beam which is used as the upper limit for data normalization of real sample images. Figure 2 a shows direct calibration images of the STEM detector and Figure $2 \mathrm{~b}$ shows reversed calibration images of the BSE detector, respectively. The bottom limit is obtained from the image captured by the BSE detector when the electron beam is blanked. It is important to take care about over/under saturation during image recording.

We proofed this method on series of samples with different composition ( $\mathrm{Cr}, \mathrm{Mo}, \mathrm{Au})$ and thicknesses. In the case of $\mathrm{Cr}$ and Mo the range was from $1 \mathrm{~nm}$ up to $25 \mathrm{~nm}$ and from $1 \mathrm{~nm}$ up to $13 \mathrm{~nm}$ in the case of Au. All samples were prepared by calibrated sputtering on a silicon wafer as a substrate. We found high precision of estimation with an error lower than $10 \%$ in most cases. The error is increasing at low thickness layers up to $70 \%$ in the case of $1 \mathrm{~nm} \mathrm{Au}$. We suggest that real error is different because of the inaccuracy of thickness determination by measuring the sputtering time. Sputtering speed was estimated by known time of sputtering and thickness measurement of the resulting layer by a profilometer. 
We assume that the presented calibration method for retractable BSE detectors can extend batch of available techniques for surface characterization of samples. The great advantage of this method is its magnification independency in a wide range from a hundred to several hundred thousand times [7].

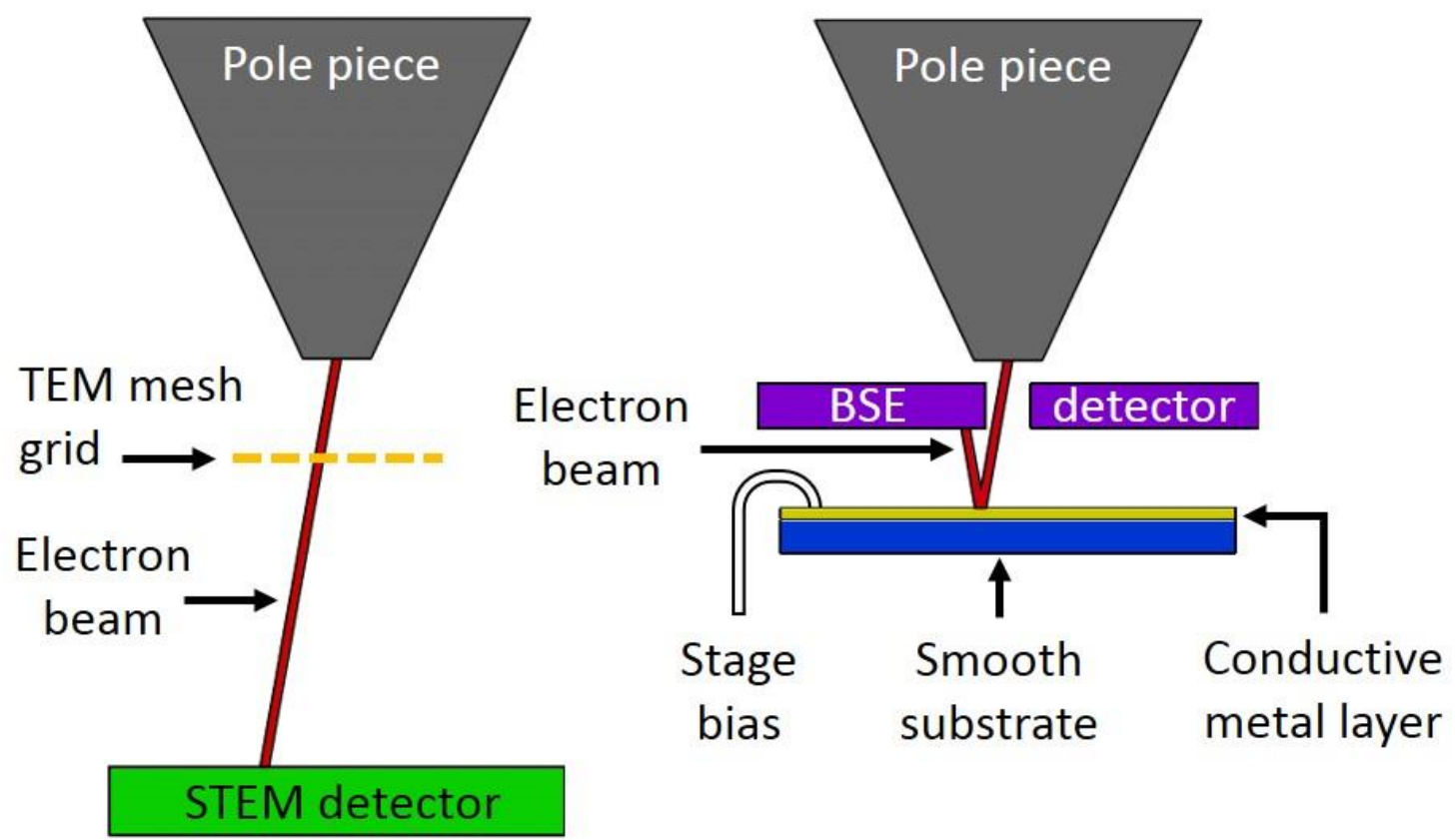

Figure 1. Diagram of energy-dependent calibration of STEM detector (left) and BSE detector (right). Calibration of BSE detector is performed by primary beam reflection on the biased sample instead of direct imaging used in the case of the STEM detector.

(a)

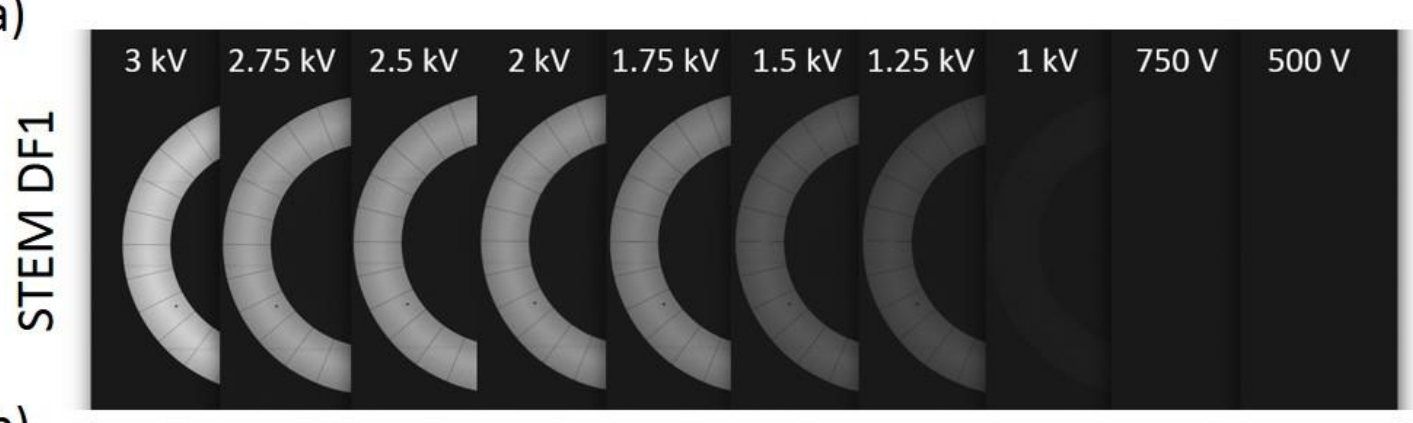

(b)

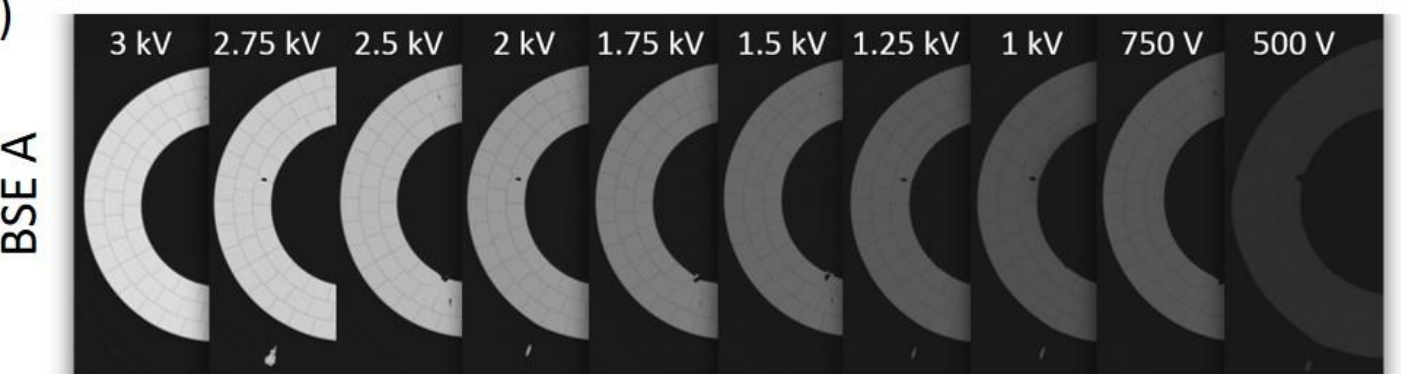

Figure 2. Dependence of pixel intensity of the inner dark field segment DF1 of the STEM detector (a) and inner BSE detector segment A (b) on beam energy. Contrast/brightness settings were the same for the individual detectors across all the energies. 
References

[1] R. Skoupy, J. Nebesarova, M. Slouf, V. Krzyzanek, Quantitative STEM imaging of electron beam induced mass loss of epoxy resin sections, Ultramicroscopy. 202 (2019) 44-50. doi:10.1016/j.ultramic.2019.03.018.

[2] M. Dapor, N. Bazzanella, L. Toniutti, A. Miotello, M. Crivellari, S. Gialanella, Backscattered electrons from gold surface films deposited on silicon substrates: A joint experimental and computational investigation to add new potentiality to electron microscopy, Surf. Interface Anal. 45 (2013) 677-681. doi:10.1002/sia.5144.

[3] J.L. Colaux, C. Jeynes, High accuracy traceable Rutherford backscattering spectrometry of ion implanted samples, Anal. Methods. 6 (2014) 120-129. doi:10.1039/c3ay41398e.

[4] A. Piegari, E. Masetti, Thin film thickness measurement: A comparison of various techniques, Thin Solid Films. 124 (1985) 249-257. doi:10.1016/0040-6090(85)90273-1.

[5] Z. Libo, B. Shengxiang, W. Rong, L. Shilan, M. Lili, L. Dechun, Thin film thickness measurement using electron probe microanalyzer, 2009 Int. Conf. Appl. Supercond. Electromagn. Devices, ASEMD 2009. (2009) 142-144. doi:10.1109/ASEMD.2009.5306671.

[6] R. Skoupy, T. Fort, V. Krzyzanek, Nanoscale Estimation of Coating Thickness on Substrates via Standardless BSE Detector Calibration, Nanomaterials. 10 (2020). doi:10.3390/nano10020332.

[7] The authors gratefully acknowledge funding from the Technology Agency of the Czech Republic (project TN01000008), the Ministry of Industry and Trade of the Czech Republic (project TRIO FV30271); the infrastructure by the Czech Academy of Sciences (project RVO:68081731). 